\title{
Detección de Thaumastocoris peregrinus (Hemiptera: Thaumastocoridae) asociado a Eucalyptus spp. en Chile
}

\author{
Detection of Thaumastocoris peregrinus (Hemiptera: Thaumastocoridae) associated \\ to Eucalyptus spp. in Chile
}

\author{
Sandra Ide $M^{\text {a }}$, Cecilia Ruiz G ${ }^{\text {b* }}$, Ariel Sandoval C ${ }^{\text {a }}$, Juan Valenzuela E ${ }^{a}$ \\ a Servicio Agrícola y Ganadero, División Protección Agrícola y Forestal, Subdepartamento Vigilancia \\ y Control Oficial Forestal, Santiago, Chile. \\ *Autor de correspondencia: ${ }^{\mathrm{b}}$ Universidad Austral de Chile, Facultad de Ciencias Forestales y Recursos Naturales, \\ Instituto de Silvicultura, Valdivia, Chile, tel.: 56-63-293085, ceciliaruiz@uach.cl
}

\begin{abstract}
SUMMARY
The presence of the bronze bug Thaumastocoris peregrinus was first reported in the country in June 2009, as a result of the Forest Surveillance Program of Servicio Agrícola y Ganadero (SAG). Sucking insects which cause damage as nymphs and adults feeding of sap on the leaves of their hosts. When populations increase, there is a sharp reduction in leaf photosynthetic rate, which can cause decreased growth and sometimes result in complete defoliation of the tree. The aims of this study were to confirm the detection of T. peregrinus, define its range and set the host range within the genus Eucalyptus species present in Chile. Thus, sticky yellow traps were installed in the metropolitan region, municipality of Til-Til, and in the region of Valparaíso; furthermore, national surveys were conducted covering the regions where species of Eucalyptus are grown. Nine positives (= presence) were detected in the metropolitan area and one in the region of Valparaíso. The detection of immature and adult T. peregrinus on Eucalyptus camaldulensis indicates that the insect is established in the region. We present actions to be carried out within the official control tasks performed by SAG.
\end{abstract}

Key words: Eucalyptus, Thaumastocoris, Eucalyptus bronze bug.

\section{RESUMEN}

La presencia del chinche del eucalipto, Thaumastocoris peregrinus, fue reportada por primera vez en el país en junio de 2009, como resultado del Programa de Vigilancia Forestal del Servicio Agrícola y Ganadero (SAG). Insecto succionador cuyo daño lo ocasionan tanto ninfas como adultos al alimentarse de la savia de sus hospederos desde las hojas. Cuando las poblaciones aumentan, se produce una fuerte reducción de la tasa fotosintética de las hojas, lo que puede provocar una disminución del crecimiento y en ocasiones producir la defoliación completa del árbol. Los objetivos de este estudio fueron constatar la detección de T. peregrinus, delimitar su área de distribución y establecer el rango de hospederos dentro de las especies del género Eucalyptus presentes en Chile. Para esto se instalaron trampas amarillas pegajosas en la región Metropolitana, comuna de Til-Til, y en la región de Valparaíso y se realizó una prospección nacional que abarcó las regiones con presencia de especies del género Eucalyptus. Se detectaron nueve puntos positivos (= presencia) en la región Metropolitana y uno en la región de Valparaíso. La detección de estados inmaduros y adultos de T. peregrinus sobre E. camaldulensis indica que el insecto se encuentra establecido en las regiones Metropolitana y de Valparaíso, Chile. Se dan a conocer las acciones a seguir dentro de las labores de control oficial ejecutadas por el SAG.

Palabras clave: Eucalyptus, Thaumastocoris, chinche del eucalipto.

\section{INTRODUCCIÓN}

Thaumastocoris peregrinus (Hemiptera: Thaumastocoridae) (Carpintero y Dellapé 2006), conocido con el nombre común de chinche del eucalipto, es un insecto fitófago de 2 a $4 \mathrm{~mm}$ de longitud, de comportamiento gregario en donde adultos y ninfas conviven en las mismas hojas y se alimentan succionando savia (Hartley 2008, Wilcken 2008, Nadel et al. 2009, Martínez et al. 2009a, Ramanagouda et al. 2010). Según Jacobs y Neser (2005), el ciclo de desarrollo de huevo a adulto, es relativamente breve, aproximadamente de 35 días, pudiendo variar según las condiciones climáticas. Sin embargo Noack y Rose (2007) señalan que en condiciones de laboratorio $\left(17\right.$ a $\left.20^{\circ} \mathrm{C}\right)$ la duración del ciclo sería de 20 días. Cada hembra puede oviponer alrededor de 60 huevos de forma agregada a lo largo de su vida (30 días) (Noack y Rose 2007). Por otra parte, el aumento de las densidades poblacionales del chinche trae consigo un aumento en el daño que ocasionan en el follaje, lo cual se traduce en una fuerte reducción de 
la tasa fotosintética, incluso la defoliación completa del árbol (Noack y Coviella 2006, Nadel et al. 2009, Martínez et al. 2009a). Este chinche se ha reportado sobre diversas especies del género Eucalyptus, sin embargo, las más susceptibles al ataque serían E. camaldulensis Dehnh., E. tereticornis Sm., E. viminalis Labill. y E. nicholli Maiden et. Blakely (Noack y Coviella 2006, Martínez et al. 2009a).

Este insecto es originario de Australia y se ha dispersado rápidamente a lugares tan distantes como Sudáfrica (2003), Zimbawe (2004), Argentina (2005), Uruguay (2008) y Brasil (2008) (Jacobs y Neser 2005, Carpintero y Dellapé 2006, Wilcken 2008, Nadel et al. 2009, Martínez y Bianchi 2010, Wilcken et al. 2010). Inicialmente esta especie fue identificada como Thaumastocoris australicus Kirkaldy (Jacobs y Neser 2005). Al año siguiente, Carpintero y Dellapé (2006) corrigen la información y determinan que se trata de una nueva especie la cual es nominada como T. peregrinus.

Considerando que esta plaga se encuentra establecida en diferentes países del Cono Sur, principalmente en la provincia de Buenos Aires en Argentina (Carpintero y Dellappé 2006), el año 2008 el Servicio Agrícola y Ganadero de Chile realizó un análisis de riesgo de plagas, para determinar el potencial de ingreso y dispersión de este insecto. Para su elaboración se utilizó la Normativa Internacional de Medidas Fitosanitarias N ${ }^{\circ} 11$ (NIMF): "Análisis de riesgo de plagas para plagas cuarentenarias". Este análisis consideró que las zonas de mayor riesgo por los daños económicos y ambientales sobre plantaciones del género Eucalyptus serían las ubicadas entre las regiones de Valparaíso y de La Araucanía (Sandoval 2008).

Durante junio de 2009, personal del Servicio Agrícola y Ganadero colectó en una trampa amarilla ubicada en la comuna de Til-Til, región Metropolitana, algunos ejemplares de un insecto perteneciente a la familia Thaumastocoridae.

Con base en los antecedentes previamente expuestos se planteó como objetivos constatar si la detección pertenecía a la especie Thaumastocoris peregrinus, delimitar su área de distribución y establecer el rango de hospederos dentro de las especies del género Eucalyptus presentes en Chile. Asimismo, informar acerca de las acciones de control a ser implementadas por el Servicio Agrícola y Ganadero.

\section{MÉTODO}

De acuerdo a los procedimientos oficiales del Servicio Agrícola y Ganadero, derivados de la intercepción de una nueva especie en el país, el material es enviado a los laboratorios para su determinación oficial por taxónomos especialistas. Esta labor se realiza por medio de claves taxonómicas o descripciones de los agentes. Una vez corroborada la identidad de la especie, se inician las labores de vigilancia específica para determinar el establecimiento, distribución y hospederos asociados. Luego de la determinación específica del ejemplar de la familia Thaumastocoridae en la región Metropolitana (junio de 2009), se iniciaron las labores de vigilancia específica: prospección (encuesta) de verificación y prospección de delimitación.

Prospección (encuesta) de verificación. Durante julio de 2009 y a partir del lugar de detección, ubicado en el fundo Tapihue, comuna de Til-Til (Datum: WGS84 Huso: 19; coordenadas UTM: E 320431, N 6333497), se prospectó en un radio de $500 \mathrm{~m}$ desde el lugar de detección sobre todos los árboles de E. camaldulensis y E. globulus encontrados dentro de esta área. Se prospectaron árboles de diferentes dimensiones, principalmente adultos sobre $15 \mathrm{~m}$ de altura y $25 \mathrm{~cm}$ de diámetro altura de pecho (DAP). No obstante, también se consideraron árboles juveniles provenientes de regeneración natural. Los árboles fueron muestreados utilizando dos técnicas: un paraguas entomológico con el propósito de constatar la presencia de ninfas y adultos a través de golpes ocasionados sobre 12 ramas por árbol (tres por cada punto cardinal), y la corta y extracción de follaje con sospecha de la presencia de huevos del insecto. Una vez que la especie se detectó sobre un hospedero, se dio por finalizada la actividad.

Prospección de delimitación. Se realizó durante los meses de noviembre y diciembre de 2009 en las principales rutas camineras de las regiones de Valparaíso y Metropolitana. Se estableció un total de 328 puntos de prospección (punto de prospección = 1 árbol), 209 en la región Metropolitana y 119 en la región de Valparaíso. La prospección se realizó generando anillos concéntricos desde los puntos previamente identificados como positivos. Adicionalmente, en la región Metropolitana se establecieron dos rutas de monitoreo (Til-Til y Santiago), considerando los lugares de mayor riesgo (Parque Metropolitano, aeropuerto nacional e internacional y principales carreteras hacia el sur del país), donde se instalaron 30 trampas amarillas pegajosas, conformadas por un tablero de forma rectangular de $10 \times 12,5 \mathrm{~cm}$ con una de sus caras de color amarillo y con un adhesivo incoloro que cubre completamente esta cara, ubicadas a 1,8 m de altura sobre árboles adultos de Eucalyptus spp. Los árboles adultos, correspondientes a individuos aislados, alcanzaron $18 \mathrm{~m}$ de altura y DAP superior a $30 \mathrm{~cm}$. Los árboles juveniles eran cortinas cortaviento de $6 \mathrm{~m}$ de altura con DAP inferior a $10 \mathrm{~cm}$. Cuando las trampas se instalaron en bosquetes fueron puestas en los bordes de éstos, preferentemente próximas a un camino principal. Las trampas fueron retiradas y revisadas quincenalmente durante ocho meses.

Entre mediados de agosto y septiembre de 2009 se realizó además un muestreo entre la región de Arica y Parinacota y la región de Los Lagos con un total de 560 puntos de prospección establecidos en lugares prioritarios (áreas aledañas a las principales carreteras y controles fronterizos), utilizando paraguas entomológicos. La prospección abarcó las especies E. camaldulensis principalmente en la zona centro norte y E. globulus en la zona centro y sur del país. 


\section{RESULTADOS}

Por primera vez en Chile se reporta la presencia de Thaumastocoris peregrinus Carpintero y Dellapé. Caracteres morfológicos externos, propios de la especie en estado adulto, como borde anterolateral del pronoto con un tubérculo a ambos lados y tres dientes subapicales oscuros sobre el margen interno de las tibias anteriores y medias, fueron encontrados en los individuos analizados (Carpintero y Dellapé 2006) (figura 1). Para la determinación se contó además con la colaboración de un especialista ${ }^{1}$, quien revisó material fotográfico de los ejemplares encontrados en Chile ${ }^{2}$. El material fue depositado en la colección de insectos de la Unidad de Entomología del Servicio Agrícola y Ganadero.

Con respecto a la prospección (encuesta) de verificación, se comprobó la presencia de huevos, ninfas y adultos sobre hojas de E. camaldulensis (figura 1). La presencia de los tres estados de desarrollo implica que el insecto logró reproducirse y que está actualmente establecido en las regiones Metropolitana y de Valparaíso.

La prospección de delimitación mostró que $T$. peregrinus se encuentra restringido a la región Metropolitana, específicamente en la comuna de Til-Til. En esta región se determinó la presencia de la plaga en nueve de las 209 estaciones de muestreo. En tanto, en la región de Valparaíso sólo una de las 119 estaciones resultó positiva, localizada en la comuna de Rinconada de Los Andes (cuesta de Chacabuco), y se detectó sólo un árbol de E. camaldulensis con presencia del insecto (figura 2). En el resto del país no se detectó la presencia de $T$. peregrinus.

Con respecto a su asociación con las especies de $E u$ calyptus, sólo se encontró en E. camaldulensis y en una

${ }^{1}$ Dr. Diego L. Carpintero de la Universidad Nacional de la Plata, Argentina.

${ }^{2}$ Patricia Jiménez Guarda. Laboratorio de Entomología. Subdepartamento Laboratorios y Estación Cuarentenaria Agrícola. Servicio Agrícola y Ganadero. Carretera 68, km 22, Lo Aguirre, Santiago, Chile.

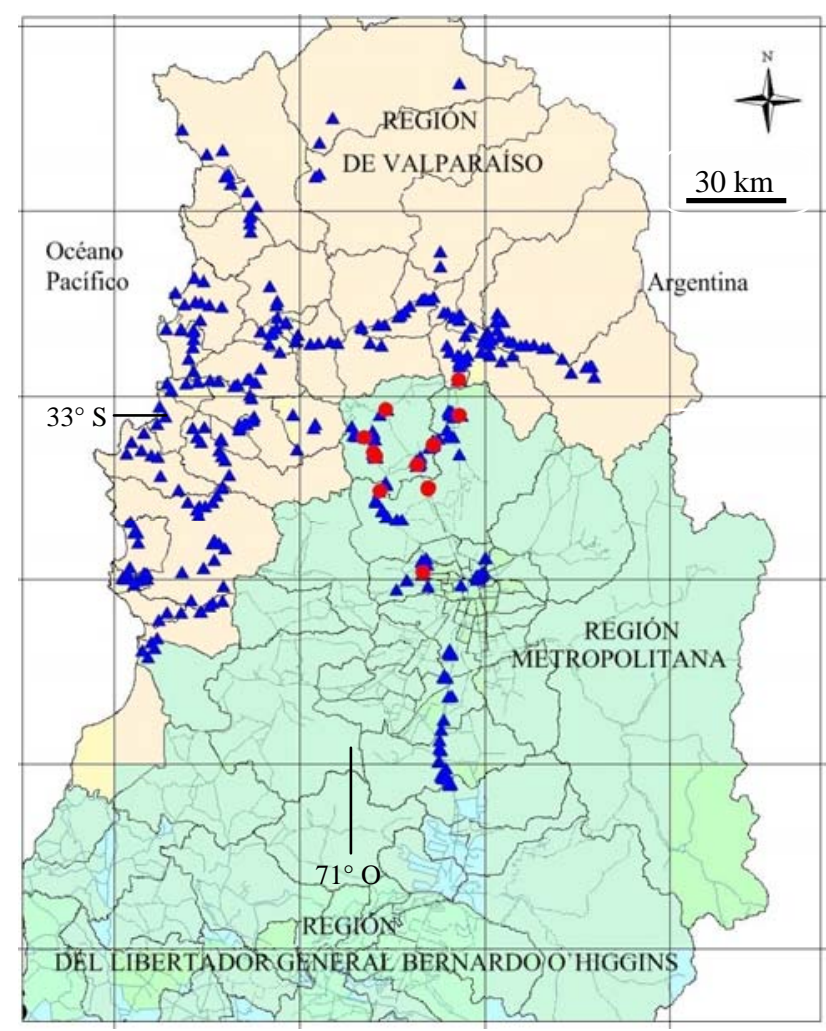

Figura 2. Mapa del área de estudio, puntos de prospección y detecciones positivas de T. peregrinus en la región de Valparaíso y Metropolitana (elaborado por el Servicio Agrícola y Ganadero de la región Metropolitana, marzo de 2011). $\bigcirc$ Presencia positiva de T. peregrinus; $\Delta$ presencia negativa de $T$. peregrinus.

Map of the study area, prospecting point and positive detections of T. peregrinus in Valparaíso and Metropolitan region (developed by Servicio Agrícola y Ganadero of the Metropolitana region, March 2011). $\bigcirc$ Positive presence of $T$. peregrinus; $\Delta$ negative presence of T. peregrinus.
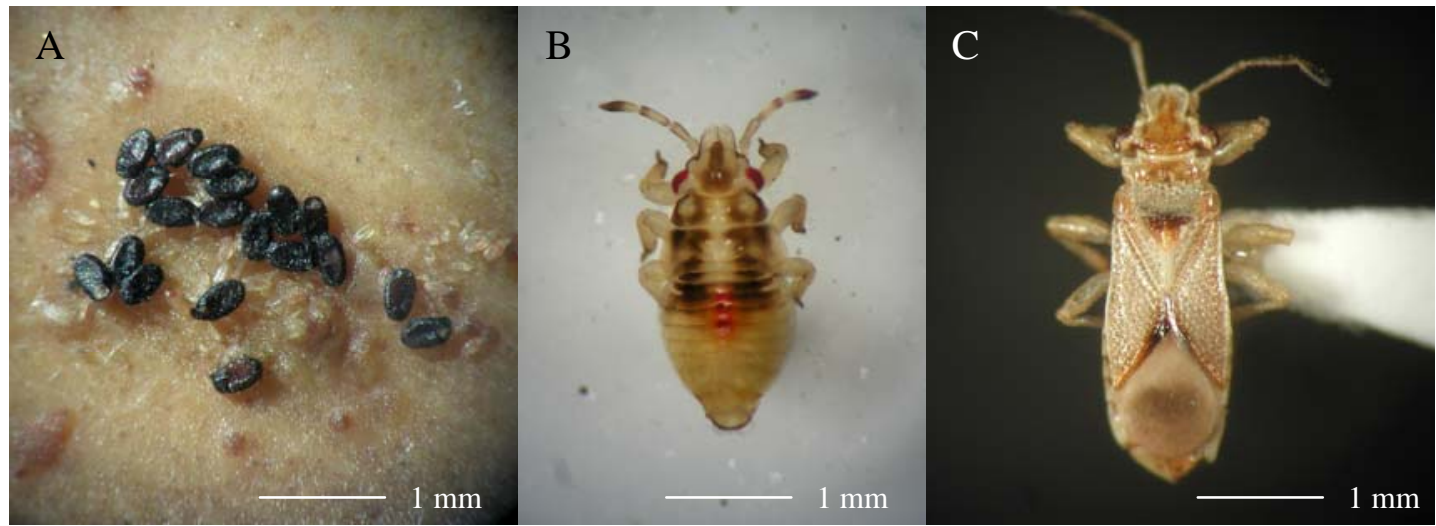

Figura 1. A) Huevos, B) ninfa y C) adulto de Thaumastocoris peregrinus.

A) Eggs, B) nymph, C) adult of Thaumastocoris peregrinus. 
oportunidad se detectaron adultos en un árbol de E. globulus. Cabe destacar que a un año de la primera detección, sólo ha sido encontrado asociado a formaciones aisladas y cortinas cortaviento, mayoritariamente de E. camaldulensis y no en plantaciones comerciales de E. globulus ni E. nitens.

Observaciones en terreno reportan además que los árboles con presencia de $T$. peregrinus también presentan ataque de Glycaspis brimblecombei (Hemiptera: Psyllidae) y se ha observado que la hembra de T. peregrinus coloca sus huevos en los bordes de los lerps de G. brimblecombei.

\section{DISCUSIÓN}

Las primeras detecciones de este insecto fuera de su lugar de origen ocurrieron en Sudáfrica y Argentina, países que se encuentran muy distantes de Australia, por lo que estos insectos pueden sobrevivir en condiciones de transporte, arribando en forma viable a nuevas zonas geográficas. En el caso específico de Chile y debido a que se presume que su dispersión es mayoritariamente pasiva, es decir, utilizando medios que le permitan transportarse, se hace indispensable el reforzamiento de las zonas fronterizas del país para evitar su dispersión principalmente a través de medios de transporte como camiones, buses, barcos, entre otros. Considerando lo anterior, Noack y Rose (2007) señalan que incluso este insecto podría asociarse a diferentes vectores, especialmente aves. Por otra parte, T. peregrinus se desarrolla en el follaje de sus especies hospederas, por lo que además, pueden ser movilizados en plantas, estacas, ramas u otro material que presente follaje.

En Argentina y Uruguay la plaga ha causado preocupación en entidades públicas y privadas, las que actualmente están trabajando en acciones de vigilancia, realizando prospecciones y monitoreos a través de trampas. Esto con la finalidad de estudiar su dispersión y las fluctuaciones poblacionales del insecto, determinándose que la plaga ha aumentado sus niveles poblacionales en forma progresiva (Martínez et al. 2009a). Esto da cuenta de su gran capacidad invasora sumada a la ausencia de enemigos naturales que pudieran controlar las poblaciones (Jacobs y Neser 2005, Carpintero y Dellape 2006). Por otra parte en Sudáfrica plantaciones comerciales del género Eucalyptus spp., han sido fuertemente afectadas por T. peregrinus (Jacobs y Neser 2005). Cabe destacar que en Australia T. peregrinus ha causado daños importantes en el arbolado urbano de Sydney en E. scoparia y E. nicholii. En este sentido Noack et al. (2009) señalan que ha tenido un efecto "devastador", situación que resulta anormal considerando que $T$. peregrinus es originario de ese país, donde debería estar en equilibrio con su medio ambiente y en especial con sus controladores biológicos.

Considerando lo anterior, es de vital importancia mantener un monitoreo constante en el tiempo, utilizando trampas para estudiar las fluctuaciones poblacionales y su relación con las condiciones ambientales. En este sentido,
Martínez et al. (2009b) proponen que el monitoreo de las poblaciones de T. peregrinus debiera efectuarse sobre los cinco metros de altura en los árboles, considerando que existen diferencias significativas en cuanto a las capturas en los distintos estratos. Sin embargo, en terreno, esto resulta muy complejo por la dificultad de acceder con la trampa a la altura sugerida. Por lo tanto, se recomienda tener en cuenta como método complementario de muestreo, implementando el corte de ramas desde el follaje superior.

A la fecha, la mayor parte de las detecciones han sido realizadas sobre E. camaldulensis, sin embargo, no se ha observado la agresividad señalada para esta plaga en otros países (Jacobs y Naser 2005, Martínez et al. 2009a, Wilcken et al. 2010). El efecto conjunto que ejercen G. brimblecombei y T. peregrinus en los árboles ha sido la clorosis o amarillamiento de las hojas, no observándose la coloración bronceada producida en ataques de la plaga en otros países donde está presente, como Uruguay, Argentina y Sudáfrica (Jacobs y Neser 2005, Nadel et al. 2009, Martínez y Bianchi 2010). Esto podría deberse a que T. peregrinus se encuentra recientemente establecido en el país y que probablemente densidades poblacionales mayores a las reportadas hasta el momento podrían provocar ese tipo de sintomatología en los árboles.

Con respecto a la disponibilidad de E. camaldulensis, una de las especies más susceptibles al ataque de T. peregrinus (Jacobs y Neser 2005, Martínez et al. 2009a), existen pequeñas plantaciones entre las regiones de Coquimbo y del Maule. Sin embargo, actualmente su utilización es principalmente ornamental y de protección de riberas (entre regiones de Arica y Parinacota y La Araucanía), por lo que el impacto provocado en esta especie de Eucalyptus sería fundamentalmente social (Dwyer et al. 2003, Noack y Coviella 2006). Adicionalmente, E. globulus está dentro de las especies reportadas como hospedero de esta plaga (Noack y Rose 2007), si bien es cierto no dentro de las más susceptibles al ataque, corresponde a la de mayor superficie y distribución en el país, existiendo actualmente unas 472 mil hectáreas (INFOR 2008).

Por otra parte, una vez constatada la presencia de T. peregrinus en Chile, el Servicio Agrícola y Ganadero comenzó con las labores de control oficial, emitiendo la Resolución $\mathrm{N}^{\circ} 4.798$ que declara el control obligatorio de la plaga (SAG 2009). A través de esta resolución se establecieron medidas fitosanitarias tendientes a disminuir la dispersión y posterior establecimiento hacia zonas no afectadas, especialmente entre las regiones del Maule y de La Araucanía.

Las acciones de control llevadas a cabo en Chile se han enfocado en el ingreso del parasitoide de huevos Cleruchoides noackae Lin y Huber (Hymenoptera: Mymaridae), originario de Australia. La colonización y establecimiento de este enemigo natural permitiría disminuir la densidad poblacional de $T$. peregrinus y así retardar su dispersión hacia aquellas zonas con plantaciones comerciales de E. globulus y E. nitens especialmente en las regiones del Biobío, La Araucanía, Los Ríos y Los Lagos. 


\section{REFERENCIAS}

Carpintero DL, PM Dellapé. 2006. A new species of Thaumastocoris Kirkaldy from Argentina (Heteroptera: Thaumastocoridae: Thaumastocorinae). Zootaxa 1228: 61-68.

Dwyer J, Nowak D, Noble M. 2003. Sustaining Urban Forests. Journal of Arboriculture 29(1): 49-55.

Hartley M. 2008. Thaumastocoris, The bronzing bug. Fact Sheet, The Arborist Network. Consultado 1 jul. 2011. Disponible en http://www.arboristnetwork.com.au/Fact Sheets.html

INFOR (Instituto Forestal, CL). 2008. Estimación de la superficie de bosques plantados por especie según región a diciembre de 2008 (ha). Consultado 7 jun. 2010. Disponible en http:// www.infor.cl/es/series-y-estadisticas/110-recurso.html

Jacobs DH, S Neser. 2005. Thaumastocoris australicus Kirkaldy (Heteroptera: Thaumastocoridae): a new insect arrival in South Africa, damaging to Eucalyptus trees. South African Journal of Science 101(5): 233-236.

Nadel RL, B Slippers, MC Scholes, SA Lawson, AE Noack, CF Wilcken, JP Bouvet, MJ Wingfield. 2009. DNA bar-coding reveals source and patterns of Thaumastocoris peregrinus invasions in South Africa and South America. Biological Invasions 12(5): 1067-1077.

Noack AE, CE Coviella. 2006. Thaumastocoris australicus Kirkaldy (Hemiptera: Thaumastocoridae): first record of this invasive pest of Eucalyptus in the Americas. General and Applied Entomology 35: 13-14.

Noack AE, HA Rose. 2007. Life-history of Thaumastocoris peregrinus and Thaumastocoris sp. in the laboratory with some observations on behaviour. General and Applied Entomology 36: 27-33.

Noack AE, J Kaapro, K Bartimote-Aufflick, S Mansfield, HA Rose. 2009. Efficacy of imidacloprid in the control of Thaumastocoris peregrinus on Eucalyptus scoparia in Sydney, Australia. Arboriculture \& Urban Forestry 35(4): 191-195.
Martínez G, S Simeto, G Balmelli. 2009a. La chinche del Eucalipto en Uruguay: Panorama de la Investigación a un Año de su Detección Oficial. Revista INIA 18:33-35.

Martínez G, P Núñez, W González, F Rodríguez, M Gómez. 2009b. Distribución vertical de la chinche del eucalipto Thaumastocoris peregrinus Carpintero y Dellapé 2006 (Hemiptera; Thaumastocoridae): Resultados preliminares. In INIA eds. Jornada Técnica, Prospección Forestal. Canelones, Uruguay. p. 31-35.

Martínez G, M Bianchi. 2010. Primer registro para Uruguay de la chinche del eucalipto, Thaumastocoris peregrinus Carpintero y Dellappé, 2006 (Heteroptera: Thaumastocoridae). Agrociencia 14(1): 15-18.

Ramanagouda S, Kumari K, Vastrad AS, Goud B, Kulkarni H. 2010. Potential alien insects threatening eucalyptus plantations in India. Karnataka Journal of Agricultural Sciences 23(1): 93-96.

SAG (Servicio Agrícola y Ganadero, CL). 2009. Resolución N ${ }^{\circ}$ 4798: Declara el control obligatorio de la plaga, chinche de los Eucalyptus, Thaumastocoris peregrinus. Santiago, Chile. Diario Oficial de la República de Chile ㅇ 39.450. 56 p.

Sandoval A. 2008. Análisis del Riesgo de Plagas para Thaumastocoris peregrinus Carpintero y Dellapé, 2006 (Hemiptera, Thaumastocoridae). Servicio Agrícola y Ganadero, División Protección Agrícola y Forestal. Santiago, Chile. 16 p. (Documento Técnico)

Wilcken C. 2008. Percevejo bronzeado do eucalipto (Thaumastocoris peregrinus) (Hemiptera: Thaumastocoridae): Ameaça ás florestas de Eucalipto brasileiras. Alerta PROTEF, Instituto de Pesquisas e Estudos Florestais, Brasil. 11 p.

Wilcken C, E Soliman, L Nogueira, L Rodrigues, T Ribeiro, P Ferreira, R Rodrigues. 2010. Bronze bug Thaumastocoris peregrinus Carpintero and Dellapé (Hemiptera: Thaumastocoridae) on Eucalyptus in Brazil and its distribution. Journal of Plant Protection Research 50(2): 201-205. 
